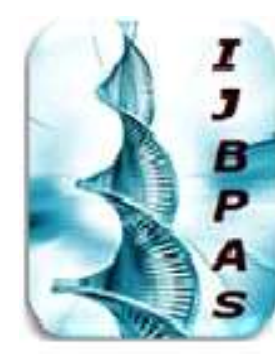

International Journal of Biology, Pharmacy and Allied Seiences (IJBPAS) 'A Bridge Betuen Caboratory and Qnendo'

WwW.ijbpas.com

\title{
A REVIEW ON FORMULATION AND EVALUATION OF SUBLINGUAL TABLETS USING SUBLIMATION TECHNIQUE
}

\author{
GADGE A*, MANTRY S AND DAMA G \\ Sharadchandra Pawar College of Pharmacy, Otur, Pune, Maharashtra, India \\ *Corresponding Author: Atul Gadge: E Mail: atulgadge4545@gmail.com
}

Received $19^{\text {th }}$ April 2021; Revised $20^{\text {th }}$ June 2021; Accepted $29^{\text {th }}$ July 2021; Available online $1^{\text {st }}$ Oct. 2021

https://doi.org/10.31032/IJBPAS/2021/10.10.1004

\begin{abstract}
Medication conveyance through the oral mucous layer is viewed as a promising option in contrast to the oral course. Sublingual course is a valuable when fast beginning of activity is wanted with preferable patient consistence over orally ingested tablets. As far as penetrability, the sublingual space of the oral pit (for example the floor of the mouth) is more penetrable than the buccal (cheek) region, which thus is more porous than the palatal (foundation of the mouth) region. The bit of medication retained through the sublingual veins side-step the hepatic first-pass metabolic cycles giving adequate bioavailability. Advantages of sublingual defeat are quick beginning of activity, patient consistence, self-sedated. Dysphagia (trouble in gulping) is normal among in all periods of individuals and more in pediatric, geriatric, mental patients. Sublingual medication organization is applied in field of cardiovascular medications, steroids, compounds and a few barbiturates. This review gives data about benefits, weaknesses, diverse sublingual definition, for example, tablets, film and their boundary.
\end{abstract}

Keywords: Sublingual delivery, Sublingual formulations, Bioavailability

\section{INTRODUCTION}

They are to be put under the tongue and produce quick foundational result by empowering the medication assimilated straightforwardly through mucosal covering of the mouth underneath the tongue. The medication retained from stomach goes to mesenteric dissemination which associates with stomach by means of entry vein. In this manner, assimilation through oral hole evades first-pass digestion. The tablets are normally little and level, packed gently to keep them delicate. The tablet should break 
up rapidly permitting the API to be consumed rapidly. It's intended to break down in little amount of spit. The medication solutes are quickly assimilated into the reticulated vein which lies under the oral mucosa, and moved through the facial veins, inside jugular vein, and braciocephalic vein and afterward depleted in to fundamental circulation [1].

The principle system for the assimilation of the medication in to oral mucosa is through detached dissemination into the lipoid layer. The retention of the medication through the sublingual course is 3 to multiple times more prominent than oral course and is just outperformed by hypodermic infusion. For these details, the little volume of spit is typically adequate to bring about tablet crumbling in the oral cavity. Sublingual items have been created for various signs going from headaches (for which fast beginning of activity is critical) to psychological instability (for which patient consistence is Important for treating ongoing signs like gloom and schizophrenia [2].

\section{SUBLINGUAL GLANDS}

Salivary organs which are available in the floor of the mouth under the tongue. They are likewise known as sublingual organs. They produce mucin thusly creates spit. The inside space of the mouth remains greased up due to production of the spit by the organs, which is important for biting and food gulping. The liquid which is created by the organs gets blend in with the food, so the food gets easily bit. Because of low emission of the salivation it can make issue in gulping the food and potential for food stop in the throat increments.

Retention implies move of medication from its site of organization to the fundamental flow, so clearly assimilation is straightforwardly corresponding to the film layer thickness. Sublingual $>$ Buccal $>$ Gingival > Palatal having mucosal thickness of 100-200, 200, 250 500-600 micrometer separately. Due to the high penetrability and the rich blood supply, the sublingual course is fit for creating a fast beginning of activity which makes it a suitable course for drugs with short conveyance period [3].

\section{COMPONENTS OF SUBLINGUAL ABSORBTION}

The ingestion of oral mucosa is impacted by the lipid solubility and consequently the porousness of the arrangement (osmosis); the ionization $(\mathrm{pH})$; and the sub-atomic load of the substances. For example, ingestion of certain medications through oral mucosa is shown to increase when transporter $\mathrm{pH}$ is bringing down (more acidic) and decrease with a bringing down of $\mathrm{pH}$ (more alkaline). 
The cells of the oral epithelium and epidermis are additionally skilled of absorbing by endocytosis (the take-up of particles by a phone as though by hollowly folding itself over it. These inundated particles are usually too huge to even think about diffusing through its divider). It is improbable that this mechanism is utilized across the whole separated epithelium. It is also unlikely that dynamic vehicle measures work inside the oral mucosa. Nonetheless, it is accepted that acidic incitement of the salivary organs, with the going with vasodilation, facilitates absorption and takeup into the circulatory framework. The mouth is lined with a mucous layer which is covered with squamous epithelium and contains mucous organs. The sublingual mucosal tissue to that of buccal mucosa.

The salivary organs comprise of lobules of cells which discharge saliva through the salivary channels into the mouth. The three sets of salivary glands are the parotid, the submandibular and the sublingual which lies on the floor of the mouth. The more corrosive the taste, the greater the incitement of salivary yield; serving to keep away from potential damage to acid-sensitive tooth finish by washing the mouth in copious neutralizing fluid. The sublingual conduit makes a trip forward to the sublingual organ, it supplies the organ and branches to the adjoining muscles and to the mucous layers of the mouth, tongue and gums. Two symmetrical branches travel behind the jawbone under the tongue to meet and join at its tip. Another branch meets and dissects with the submental branches of the facial conduit. The sublingual conduit comes from the lingual course - the body's main blood supply to the tongue and the floor of the mouth - which emerges from the outside carotid corridor. The proximity with the inside carotid course permits quick admittance to its route supply most of the cerebral side of the equator [4].

\section{MECHANISM FOR SUBLINGUAL DRUG DELIVERY SYSTEMS}

Sublingual medications are placed under the tongue. They dissolve there, and their active ingredients are quickly absorbed into the bloodstream. There are tablets, spray, and film sublinguals. Administration through direct absorption into the mouth provides an advantage to medications you swallow.

\section{MEDICATIONS FOR SUBLINGUAL ADMINISTRATION}

Sublingual medication organization is applied in the field of cardiovascular medications, steroids, some barbiturates and compounds. It has been a developing field in the organization of numerous nutrients and 
minerals which are observed to be promptly and thoroughly consumed by this technique. Sublingually absorbed nourishment, which dodges openness to the gastric framework and liver, implies direct healthful advantages, especially significant for victims of gastro-intestinal hardships like ulcers, hyperactive gut, coeliac illness, those with compromised assimilation, the old and invalids - the wholesome advantage is free of gastro-intestinal impacts. Instances of drugs administered by this course incorporate antiangina like nitrites and nitrates, against hypertensive like nifedipine, analgesics like morphine and bronchodilators like fenoterol. Certain steroids like estradiol and peptides like oxytocin can likewise be administered for example fentanyl citrate, apomorphine, prochlorperazinedimaleate $\{\mathrm{PRO}\}$, and hydrazine $\mathrm{HCl}$.

\section{BENEFITS}

Good mouth feels property assists with changing the basic perspective taking drugs as "unpleasant reality", especially for pediatric patients.

Fast disintegration of medicament and absorption which will prompts fast, beginning of activity.

Some drugs are retained from the mouthpharynx and throat as the salivation passes down into the stomach, in such cases bioavailability of medications is expanded.

It gives benefits of fluid formulation in the type of strong measurements structure.

$>$ Pregastric retention can bring about improved bioavailability and because of decreased measurements, worked on clinical execution through a Reduction of undesirable impact.

\section{DRAWBACKS}

Since sublingual organization of medications meddles with eating, drinking, and talking, this course is by and large viewed as un reasonable for delayed organization.

$>$ Although this site isn't appropriate to sustained-delivery frameworks.

> Sublingual prescription cannot be utilized when a patient is uncooperative or oblivious.

The patient ought not smoke while taking sublingual prescription, since smoking causes vasoconstriction of the veins. This will diminish the retention of the medication [5].

\section{SUBLINGUAL FORMULATIONS SUBLINGUAL TABLETS}

They are to be set under the tongue and produce quick fundamental outcome by empowering the medication assimilated 
straightforwardly through mucosal covering of the mouth underneath the tongue. The medication assimilated from stomach goes to mesenteric course which associates with stomach by means of gateway vein. Consequently, retention through oral pit keeps away from first-pass digestion. The tablets are typically little and level, packed daintily to keep them delicate. The tablet should break up rapidly permitting the API to be ingested rapidly. It's intended to break down in little amount of salivation. After the tablet is put in the mouth underneath the tongue, the patient ought to abstain from eating, drinking, smoking and potentially talking to keep the tablet set up. Gulping of spit ought to likewise be stayed away from since the salivation might contain broke up drug. Tasteless excipients are utilized to stay away from salivary incitement.

Some drugs goes through broad first pass digestion which brings about helpless bioavailability of its oral measurement shapes that sort of medications are appropriate for sublingual dose structure. Drugs that are shaky in parenteral readiness are appropriate for sublingual measurement structure [6].

QUICK DISINTEGRATING SUBLINGUAL TABLETS
Tablets that crumble or disintegrate quickly in the patient's mouth are advantageous for small kids, the older and patients with gulping hardships, and in circumstances where consumable fluids are not accessible. Just the little volume of spit is generally adequate to bring about tablet deterioration in the oral hole. The prescription would then be able to be ingested to some degree or completely into the fundamental dissemination from veins in the sublingual mucosa. The sublingual course typically creates a quicker beginning of activity than orally ingested tablets and the bit consumed through the sublingual veins sidesteps the hepatic first-pass metabolic cycles.

\section{FORMULATION AND DEVELOPMENT}

To formulate rapidly-disintegrating or dissolving tablets. Direct compression, one of these techniques, requires the incorporation of a superdisintegrant into the formulation, or the use of highly water-soluble excipients to achieve fast tablet disintegration. Direct compression does not require the use of water or heat during the formulation procedure and is the ideal method for moisture- and heat-labile medications. However, the direct compression method is very sensitive to changes in the type and proportion of excipients and in the compression forces, when used to achieve 
tablets of suitable hardness without compromising the rapid disintegration characteristics. Extremely fast tablet disintegration would be required to enhance the release of drug from tablets for rapid absorption by the sublingual mucosa blood vessels [7].

\section{Bioadhesive sublingual tablets}

The new sublingual tablet concept presented is based on interactive mixtures consisting of a water soluble carrier covered with fine drug particles and a bioadhesive component. With this approach, it is possible to maintain rapid dissolution in combination with bioadhesive retention of the drug in the oral cavity. Bioadhesion is usually defined as the bond formed between two biological surfaces or between a biological and a synthetic surface. Problem associated with sublingual tablet formulation is that there is always a risk that the patient will swallow part of the dose before the active substance has been released and absorbed locally into systemic circulation. This could result an unwanted prolongation of the pharmacological effect. Addition of a bioadhesive component is a well-known method of increasing the possibility of a more site-specific release. However, this conceptis normally applied to non-disintegrating tablets or disc to achieve extended release of the active substance and, consequently, such a system will not be suitable for a fast acting formulation. Therefore, it would be of interest to study a disintegrating tablet which releases the drug quickly, but which also has bioadhesive properties which could prevent the drug from being swallowed [8].

\section{Bioadhesion mechanisms}

The mucus layer is often involved in the adhesion of a bioadhesive polymer and is present as either a gel layer adhering to the mucosal surface or a solution or suspension of various substances. The mucus layer mainly consists of mucin glycoproteins, inorganic salts, proteins, lipids and water, with the composition varying depending on its source. The electronic theory involves an electronic transfer between the two materials causing a double layer of electrical charge, which results in attraction forces. The adsorption theory involves adhesion between the mucosa and the adhesive material by van der Waals interactions, hydrogen bonds and related forces. The wetting theory involves interfacial tensions between the two materials. Penetration of polymer chains into the mucus network and vice versa, causing a mechanical bond, is referred to as the diffusion theory. The importance of water content and movement of water into the bioadhesive material from the mucosa, i.e. 
dehydration of the mucosa, has also been suggested as a mechanism for adhesion [9].

\section{Measurement of bio-adhesive strength}

Bio-adhesive strength of the tablets was measured on a modified physical balance using the method described by Gupta et al. The method used bovine cheek pouch as the model mucosal membrane and IPB pH 6.6 as the moistening fluid. The surface of the mucosal membrane was first blotted with a filter paper and then moistened with $25 / \mathrm{L} 1$ of IPB $\mathrm{pH}$ 6.6. The weight, in required to detach the tablets from the mucosal surface gave the measure of bio-adhesive strength [10].

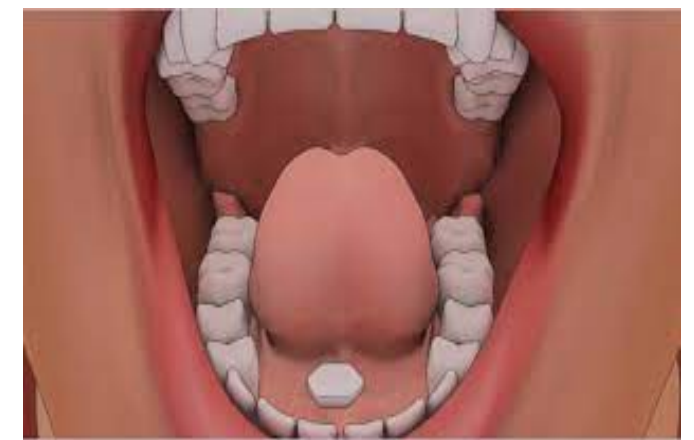

Figure 1: Sublingual Route of Administration (Source: Google Image)

\section{THIN FILM DRUG DELIVERY}

Thin film drug delivery is a process of delivering drugs to the systemic circulation via a thin film that dissolves when in contact with liquid, often referred to as a dissolving film or strip. Thin film strips are typically designed for oral administration, with the user placing the strip on or under the tongue or along the inside of the cheek. As the strip dissolves, the drug can enter the blood stream enterically, buccally or sublingually. The sublingual delivery of a drug via thin film has the potential to improve the onset of action, lower the dosing, and enhance the efficacy and safety profile of the medicament. All tablet dosage forms, soft gels and liquid formulations primarily enter the blood stream via the gastrointestinal tract, which subjects the drug to degradation from stomach acid, bile, digestive enzymes and other first pass effects. As a result, such formulations often require higher doses and generally have a delayed onset of action. Conversely, sublingual thin film drug delivery can avoid these issues and yield quicker onsets of action at lower doses. Thin film is more stable, durable and quicker dissolving than other conventional dosage forms. Thin film enables improved dosing accuracy relative to liquid formulations since every strip is manufactured to contain a precise amount of the drug. Thin film not only ensures more accurate administration of drugs but also can improve compliance due to the intuitive nature of the dosage form and its inherent ease of administration. These properties are especially beneficial for pediatric, geriatric and neurodegenerative disease patients where proper and complete dosing can be difficult. Thin film's ability to dissolve rapidly without the need for water 
provides an alternative to patients with swallowing disorders and to patients suffering from nausea, such as those patients receiving chemotherapy. Thin film drug delivery has the potential to allow the development of sensitive drug targets that may otherwise not be possible in tablet or liquid formulations. From a commercial perspective thin film drug delivery technology offers an opportunity to extend revenue lifecycles for pharmaceutical companies whose drug patent is expiring and will soon be vulnerable to generic competition [11].

\section{Thin film drug delivery}

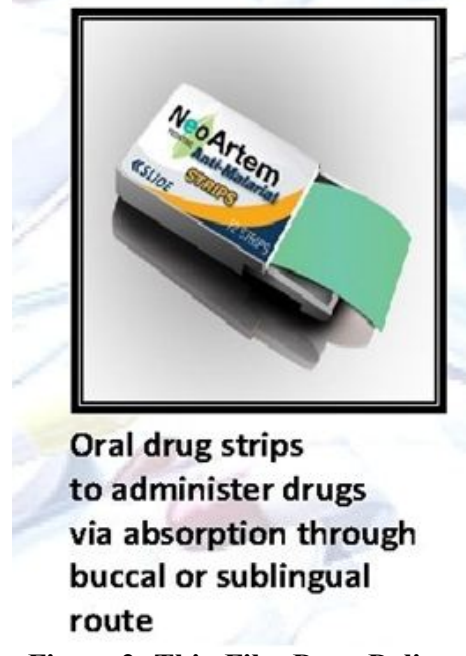

Figure 2: Thin Film Drug Delivery

\section{LIPID MATRIX SUBLINGUAL}

\section{TABLETS}

Lipid Matrix Sublingual Tablet is formulated using advances in sublingual and liposomal technology to create a dosage form that offers a faster and more complete absorption than traditional oral routes of administration. The Lipid Matrix Sublingual Tablet is a bioavailable, quick, convenient, and consistent dosage form for many specialty nutra ceuticals that are often taken orally. Examples Include: Glutathione MB12 (Methylcobalamin) Melatoni.

\section{SUBLINGUAL IMMUNOTHERAPY}

Sublingual immunotherapy, or SLIT, is a form of immunotherapy that involves putting drops of allergen extracts under the tongue. SLIT is usually delivered one of two ways: drops (or tablets) of allergen extract are placed under the tongue, then either swallowed or spat out. Most studies have looked at swallowing the extract, which seems to work better. Immunotherapy by the oral route (swallowed and not kept under the tongue for any period of time), causes too many gastrointestinal side effects (nausea, vomiting, diarrhea), and therefore is not used. Generally, SLIT is administered daily, or multiple times per week, over a period of year. Sublingual monomeric aller goid in tablets given to pediatric patients with allergic rhinitis and/or asthma patient.

\section{SUBLINGUAL VITAMIN TABLETS}

The only Sublingual vitamin that all doctors recommend is vitamin B12 (Cyanocobalamin). Recommended to be taken once a day. 


\section{EVALUATION}

\section{Hardness and thickness}

The test is done as per the standard methods. The hardness of three randomly selected tablets from each formulation is determined by placing each tablet diagonally between the two plungers of tablet hardness tester (with the nozzle) and applying pressure until the tablet broke down into two parts completely and the reading on the scale is noted down. The thickness of three randomly selected tablets from each formulation is determined in mmusing a verniercaliper (Pico India). The average values are calculated.

\section{Drug Content}

Randomly ten tablets are selected from formulation, finely powdered and powder equivalent $\mathrm{mg}$ of drug is accurately weighed andtransferred to $100 \mathrm{ml}$ volumetric flasks containing solution of desired $\mathrm{pH}$. The flask is shaken to mix the contents thoroughly. The volume is made up to the mark with solution and filtered. One $\mathrm{ml}$ of the filtrate is suitably diluted and drug content is estimated using a double beam UV-visible spectrophotometer. This procedure is repeated thrice and the average values are calculated [12].

\section{Wetting time (WT)}

It is useful for quality control and provides supportive evaluation of this sublingual tablets. Unlike the disintegration test, the wetting test uses minimal water, which may be more representative of the quantity of moisture available sublingually. Using this test, the time required for moisture to penetrate the tablet completely is measured and possibly represents the time required to release drug in the presence of minute volumes of saliva. The tablet was placed above absorbent paper fitted into a petri dish. After the paper is thoroughly wetted with distilled water, excess water is completely drained out of the dish. The time required for the water to diffuse from the wetted absorbent paper throughout the entire tablet is then recorded using a stopwatch [13].

\section{Disintegration test}

A relatively simple method with rigorous conditions is developed. Each individual tablet is dropped into $10-\mathrm{ml}$ glass test tube (1.5-cmdiameter) containing $2 \mathrm{ml}$ distilled water, and the time required for complete tablet disintegration is observed visually and recorded using a stopwatch. The visual inspection is enhanced by gently rotating the test tube at a 450 angle, without agitation, to distribute any tablet particles that might mask any remaining undisintegrated portion of the tablets. In the USP disintegration test for sublingual tablets, the disintegration apparatus for oral tablets is used without the covering plastic disks, and 2 minutes is 
specified as the acceptable time limit for tablet disintegration.

\section{In vitro disintegrating test}

A disintegration time for sublingual tablets is determined using USP tablet disintegration apparatus with desired medium. The volume of medium was $25 \mathrm{ml}$ and temp was $37 \pm 2$ ${ }^{\circ} \mathrm{C}$. The time in seconds taken for complete disintegration of the tablets with no palatable mass remaining in the apparatus is measured.

\section{In vitro dissolution test}

In vitro release rate of sublingual tablets will be carried out using United State Pharmacopoeia (USP) XXIV dissolution testing apparatus (Paddle method). An aliquot sample of the solution is withdrawn from the dissolution apparatus. The Samples are replaced with fresh dissolution medium of same quantity. The samples are filtered through Whatman filter paper No 40 and analyze din UV spectrophotometer. The percentage drug release is calculated using an equation obtained from the calibration curve [14].

\section{MARKETED PREPARATIONS}

\begin{tabular}{|c|c|}
\hline Marketed Preparations & Name of Company \\
\hline $\begin{array}{c}\text { Tenormin sublingual } \\
\text { tablet (isoproterenol) }\end{array}$ & Actavis Pharma \\
\hline $\begin{array}{c}\text { Microtab sublingual } \\
\text { tablet (nicotine) }\end{array}$ & $\begin{array}{c}\text { McNeil Products } \\
\text { Limited }\end{array}$ \\
\hline $\begin{array}{c}\text { Nascobal sublingual } \\
\text { tablet (vitamin } \text { B }_{12} \text { ) }\end{array}$ & $\begin{array}{c}\text { Endo Pharmaceuticals } \\
\text { Inc. }\end{array}$ \\
\hline $\begin{array}{c}\text { Subuter sublingual tablet } \\
\text { (buprenorphine) }\end{array}$ & $\begin{array}{c}\text { Reckitt Benckiser } \\
\text { Pharmaceuticals Inc. }\end{array}$ \\
\hline $\begin{array}{c}\text { Nitroquick sublingual } \\
\text { tablet (nitroglycerin) }\end{array}$ & $\begin{array}{c}\text { Steris Healthcare Pvt } \\
\text { Ltd }\end{array}$ \\
\hline
\end{tabular}

\section{CONCLUSION}

Sublingual drug delivery has been used for formulation of many drugs with view point of rapid drug release and quick onset of action. Sublingual products were developed to overcome the difficulty in swallowing conventional tablet, among pediatric, geriatric and psychiatric patients with dysphagia. The target population has expanded to those who want convenient dosing without water anywhere, anytime. The potential for such dosage forms is promising because strong market acceptance and patient demand. Peak blood levels of most products administered sublingually are achieved within 10-15 minutes, which is generally much faster than when those same drugs are ingested orally. Sublingual absorption is efficient. The percent of each dose absorbed is generally higher than that achieved by means of oral ingestion. Various types of sublingual dosage forms are available in market like tablets, films and sprays. This type of drug delivery is likely to be very popular because of its immediate results and palatability.

\section{REFERENCES}

[1] Narang N, Sharma J. Sublingual mucosa as a route for systemic drug delivery. Int J Pharm Pharm Sci. 2011; 3(Supp1 2): 18-22. 
[2] Chickering DE, Lehr CM,

Mathiowitz E, editors. Bioadhesive drug delivery systems: fundamentals, novel approaches, and development. Marcel Dekker; 1999.

[3] Richman MD, Fox CD, Shangraw RF. Preparation and stability of glyceryl trinitrate sublingual tablets prepared by direct compression. Journal of pharmaceutical sciences. 1965 Mar 1; 54(3): 447-51.

[4] Duchěne D, Touchard F, Peppas NA. Pharmaceutical and medical aspects of bioadhesive systems for drug administration. Drug development and industrial pharmacy. 1988 Jan 1; 14(2-3): 283318.

[5] Tur KM, Ch'ng HS. Evaluation of possible mechanism (s) of bioadhesion. International journal of pharmaceutics. 1998 Jan 12; 160(1): $61-74$

[6] Harris D, Robinson JR. Drug delivery via the mucous membranes of the oral cavity. Journal of pharmaceutical sciences. 1992 Jan 1; 81(1): 1-0.

[7] Birudaraj R, Berner B, Shen S, Li X. Buccal permeation of buspirone: mechanistic studies on transport pathways. Journal of pharmaceutical sciences. 2005 Jan 1; 94(1): 70-8.

[8] Ishikawa T, Koizumi N, Mukai B, Utoguchi N, Fujii M, Matsumoto M, Endo H, Shirotake S, Watanabe Y. Pharmacokinetics of acetaminophen from rapidly disintegrating compressed tablet prepared using microcrystalline cellulose (PH-M06) and spherical sugar granules. Chemical and pharmaceutical bulletin. 2001;49(2):230-2.

[9] Price TM, Blauer KL, Hansen M, Stanczyk F, Lobo R, Bates GW. Single-dose pharmacokinetics of sublingual versus oral administration of micronized $17 \beta$ estradiol. Obstetrics \& Gynecology. 1997 Mar 1; 89(3): 340-5.

[10] Kurosaki Y, Takatori T, Nishimura $H$, Nakayama $T$, Kimura $T$. Regional variation in oral mucosal drug absorption: permeability and degree of keratinization in hamster oral cavity. Pharmaceutical research. 1991 Oct; 8(10): 1297301.

[11] Ghosh TK, Chatterjee DJ, Pfister WR, Jarugula VR, Fadiran EO, Hunt JP, Lesko LJ, Tammara VK, 
Hare DB. Quick-Dissolving Oral

Dosage Forms: Scientific and

Regulatory Considerations from a

Clinical Pharmacology and

Biopharmaceutics Perspective.

Drugs and the Pharmaceutical

Sciences. 2005 Feb 28; 145: 337.

[12] De Boer AG, De Leede LG, Breimer DD. Drug absorption by sublingual and rectal routes. British journal of anaesthesia. 1984 Jan 1; 56(1): 69-82.
[13] Al-Ghananeem AM, Malkawi AH, Crooks PA. Effect of $\mathrm{pH}$ on sublingual absorption of oxycodone hydrochloride. Aaps Pharmscitech. 2006 Mar; 7(1): E163-7.

[14] Allen Jr LV. Rapid-dissolve technology: An interview with Loyd V. Allen, Jr., PhD, RPh. International Journal of Pharmaceutical Compounding2003 Nov 1; 7(6):449. 